\title{
POLARIMETRIC MINISAR MAPPING SYSTEM
}

\author{
J. X. Zhang ${ }^{1}$, L. J. Lu* ${ }^{2 *}$ G. M. Huang ${ }^{2}$, S.C.Yang ${ }^{2}$, C. Q. Cheng ${ }^{2}$, Z. Zhao ${ }^{2}$ \\ ${ }^{1}$ National Quality Inspection and Testing Center for Surveying and Mapping Products, 100830 Beijing, China - \\ zhangjixian@vip.sina.com \\ 2 Key Laboratory of Earth Observation and Geospatial Information Science, Chinese Academy of Surveying and Mapping, 100830 \\ Beijing, China \\ Information extraction, knowledge discovery and data mining techniques
}

KEY WORDS: Polarimetric, MiniSAR, UAV, mapping

\begin{abstract}
:
Polarimetric MiniSAR is a ku-band fully polarimetric SAR mapping system that is being developed at CASM (Chinese Academy of Surveying and Mapping). The system consists of a ku-band fully polarimetric miniSAR data acquisition system and a set of miniSAR mapping software. Nowadays, as the most representative SAR system in China, polarimetric miniSAR mapping system have developed multiple advanced miniSAR mapping techniques, a large scale of mapping experiment has been achieved.
\end{abstract}

\section{INTRODUCTION}

Nowadays, mapping and emergency response in cloudy and foggy areas have become a major demand for mapping in China. In the context, SAR mapping, as an effective mapping technology, has been playing an increasingly plays an increasingly significant role. As the core instrument, SAR sensor has greatly improved the performance, small and lightweight sensor is latest development trend. MicroASAR is small, low-cost, SAR systems designed by BYU (Brigham Young University) Artemis Inc. and Hauppauge (Edwards et al., 2008). The MicroASAR has been designed specifically to be small and lightweight (a mass of less than $3.3 \mathrm{~kg}$ ) while still being robust and capable. Massive high position accuracy, highresolution, multi-band and multi-polarization SAR sensors have been developed recently. SAR that represent a new advancement in high-performance SAR applications is a SlimSAR designed by the same organization as MicroASAR (Zaugg et al., 2010). The high-precision GPS receiver and an IMU (Inertial Measurement Unit) guarantees high-precision inertial measurements. In addition, multi-band ( $\mathrm{X}$ and $\mathrm{Ku}$ band) and multi-polarization (transmitting and receiving horizontal and vertical polarization) imagery can be available for SlimSAR system. MiniSAR systems has been applied in topographic mapping and emergency response, such as natural hazards and environmental monitoring in past years (Zaugg et al., 2010).

Polarimetric MiniSAR, as China's first miniSAR mapping system, has been developed by CASM. Polarimetric MiniSAR system represents the newest achievement in high-performance, small, low-cost, SAR, mapping science, suitable for operation on small UAV, satisfying the urgent needs of cloudy area mapping and emergency response. The system will be deployed as a SAR sensor for a unmanned Aerial Vehicle (UAV). Initially, the ku-band fully polarimetric MiniSAR sensor was integrated in a motorglider platform. A set of MiniSAR mapping software for 3D (DOM, DEM, and DLG) product generation has been developed by CASM. A few of applications of 3D mapping and sea ice monitoring have implemented with the Polarimetric MiniSAR system. In the fol-

\footnotetext{
* Corresponding author

Email: lulj@casm.ac.cn
}

lowing future, the UAV will provide an excellent opportunity for the demonstration and evaluation of SAR technology for unmanned monitoring and emergency response tasks.

\section{POLARIMETRIC MINISAR DATA ACQUISITION SYSTEM}

\subsection{Ku-band MiniSAR Sensor Design}

The miniSAR sensor is designed for use of a linear frequencymodulated continuous-wave (LFM-CW) signal facilitates compact design, allowing us to achieve a high signal-to-noise ratio while transmitting with less peak power. The core portions of $\mathrm{Ku}$-band sensor consisting of SAR radio frequency (RF) subsystem and digital subsystem are integrated into the enclosure, weighing only $1.8 \mathrm{Kg}$, as shown in Figure 1 . The RF subsystem was designed by using the advanced micro-assembly technology such as LTCC, and the digital subsystem was designed by using highly integrated Field Programmable Gate Array (FPGA).

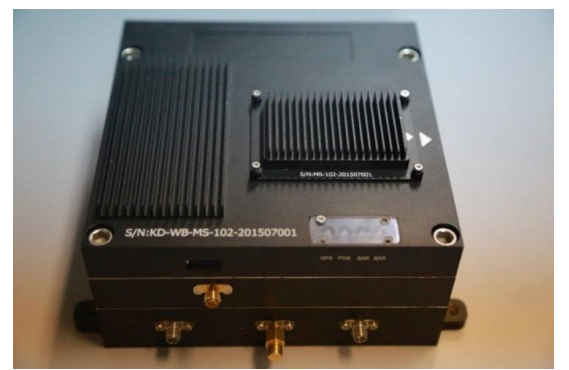

Figure 1. RF and digital subsystems integrated into the enclosure

Owing to the full polarimetric SAR system, the antenna array is composed of $4 * 24$ array elements as shown in Figure 2, and separation wall is added between transmit and receive antennas that ensure the requirements of cross polarization isolation (better than $-25 \mathrm{~dB}$ ). The technique of microstrip paster array antenna has been used in the micro high performance dual polarization antenna, and the joint scheme of slot coupling and line feeding has been adopted in the polarization coplanar 
antenna.

A flight control and monitoring subsystem is a software running

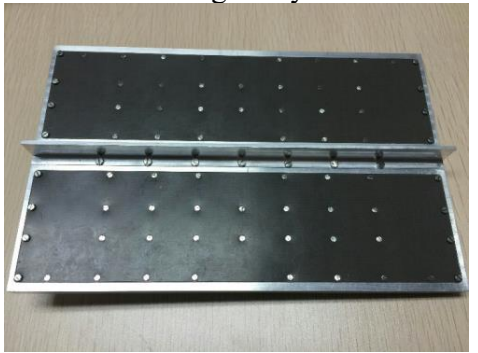

Figure 2. The antenna array of full polarimetric SAR system

on the flight control computer which is mainly used for the control and monitoring of RF and digital subsystems.

The position measurement subsystem includes miniature GPS receiver and an IMU (Inertial Measurement Unit). Aiding by the differential GPS (dGPS) base station, the position accuracy is estimated at 1 2 meter.

\subsection{Polarimetric MiniSAR Data Acquisition System Integration}

The miniSAR sensor, flight platform (UAV) and ground control system constitute polarimetric SAR data acquisition system.

\section{(1)The integration of sensor and UAV}

The selected UAV has the length of 1.87 meter, has a wingspan of 4.8 meters and has payloads of $10 \mathrm{Kg}$. The UAV cabin has been modified to accommodate the miniSAR sensor and navigation equipments with the position measurement subsystem and flight control and monitoring subsystem. Considering the effects of reflection and occlusion, radar antenna installed on the side of head of UAV, as shown in Figure 3.

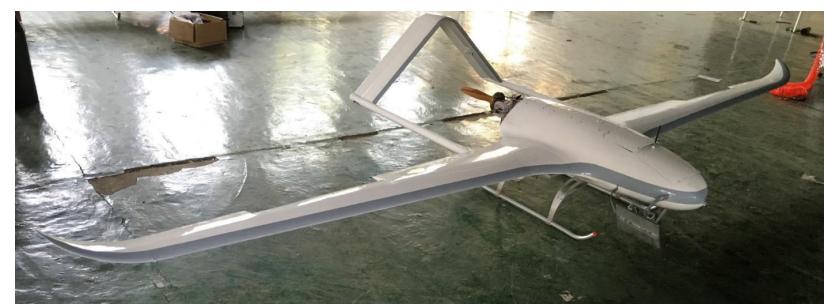

Figure 3 The outlook of UAV

(2)The integration of miniSAR sensor and ground control system

The ground control system, as shown in Figure 4, is capable of communicating with navigation equipments mounted on UAV, and has the functions of flight mission route management, precise flight route control and real-time SAR imaging.

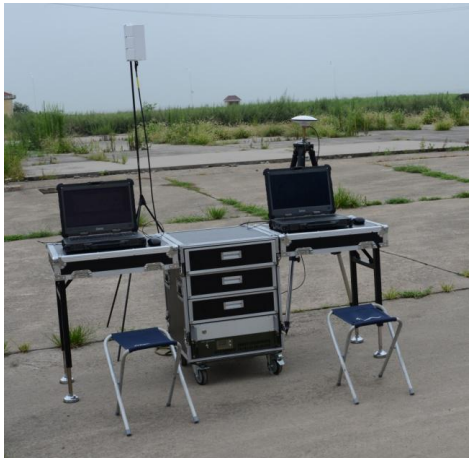

Figure 4 The ground control system

\subsection{System Specifications}

Polarimetric miniSAR data acquisition system consisting of the miniSAR sensor, flight platform (UAV) and ground control system has the following technical specification.

(1) MiniSAR sensor specifications

Table 1. Sensor parameters

\begin{tabular}{|l|l|}
\hline Operating frequency $(\mathrm{GHz})$ & 14.5 \\
\hline Range resolution $(\mathrm{m})$ & 0.2 \\
\hline Maximum Range $(\mathrm{km})$ & 6 \\
\hline Swath width $(\mathrm{km})$ & 3 \\
\hline Total weight $(\mathrm{kg})$ & $<5$ \\
\hline Power consumption $(\mathrm{w})$ & $<150$ \\
\hline Polarization mode & $\mathrm{HH}, \mathrm{HV}, \mathrm{VH}, \mathrm{VV}$ \\
\hline Polarization isolation $(\mathrm{dB})$ & $<-25$ \\
\hline Radiometric calibration accuracy $(\mathrm{dB})$ & $<1.5$ \\
\hline
\end{tabular}

(2) UAV specifications

Table 2. UAV parameters

\begin{tabular}{|l|l|}
\hline Flight altitude (m) & $100 \sim 5000$ \\
\hline Duration (hour) & $>3$ \\
\hline Payload mass(kg) & $>10$ \\
\hline
\end{tabular}

(3) Ground control system specifications

Table 3. Ground control system parameters

\begin{tabular}{|l|l|}
\hline Real-time SAR imaging (second) & $<20$ delay
\end{tabular}

\section{MINISAR MAPPING SOFTWARE}

According to the characteristics of miniSAR imagery, high frequency, high resolution, narrow swath width and relative low position accuracy, the latest MiniSAR mapping software has been developed by CASM. Several advanced techniques has been embedded in the software. These technical advancements have made it possible to optimize the processing of MiniSAR imagery for accurate product generation.

\subsection{Multi-images Block Adjustment}

Aiming at the multiple MiniSAR images processing, the block adjustment technique is applied by a universal geometric model such as a range-coplanarity model (Cheng et al., 2010) construction, improving the positioning accuracy of MiniSAR image is improved with few control points. In most cases, the plane and elevation accuracy are better than $0.3 \mathrm{~m}$ only using 14 GCPs (Ground Control Points).

\subsection{Multi-images Geometric Rectification and Mosic}

Multi-images geometric rectification processing is based on SAR imaging geometry model, in the case, the rigorous Range- 
Doppler (RD) model is used (Meier et al., 1993). For the purpose of fast multi-images geometric rectification processing, a strategy of block-based fast geometric rectification is developed, i.e., the image is segmented by appropriate block size, and then the coordinates of each pixel point in the block are calculated by indirect positioning. In the following, multiimages mosic can be implemented on the orth-rectification images. A mosaic method for SAR Images based on fast filling of scanning lines is used here to obtain accurate and highquality mosic orthophoto.

\subsection{Radargrammetry for MiniSAR}

A technique of making stereo model based on geometry transformation is used in terms of the features of miniSAR image. The technique first make the slant-ground range transforms for original SAR stereo image pair, then rotates the right image pair to eliminate the upper and down parallax to generate the ideal stereo image pair. At the same time, the measurement model is constructed according to the original SAR stereo geometric model, so that the stereo model for digital mapping is established. This technology can acquire good stereo vision, and achieve high precision stereo measurement, and be used for three-dimensional information extraction and interpretation.

\subsection{Dimensionality Reduction and Fusion of Multi- Features on Polarimetric MiniSAR Image}

For polarimetric SAR image, more than hundreds of polarimetric features can be extracted on coherent and incoherent PolSAR decomposition models. In light of the characteristics of high frequency and high resolution, miniSAR image has clear details and texture information, however, the characteristics of polarization are not significant. Therefore, plenty of polarimetric and texture features are extracted, then supervised dimension reduction method based on graph embedding theory (Shi et al., 2013) is used that can maintain polarization information and high-precision texture information, so as to enhance the ability of polarimetric miniSAR image classification.

\section{MINISAR MAPPING APPLICATION}

\subsection{D Mapping}

In 2017, 1:2000 3D mapping campaign was implemented in experimental area of Jishan county, Shanxi province, China. The experimental area has three kinds of terrain, plain, hill and mount. The whole area is covered by an amount of planted crops, buildings and rivers.

First, a mount of polarimetric miniSAR data was acquired from two direction (from west to east and from east to west), and 16 routes and 75 scenes images were collected in total. Orthophoto after images mosaic are shown in Figure 5.

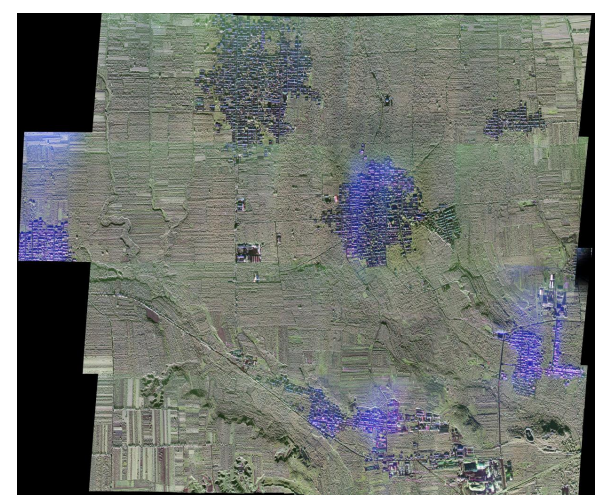

Figure 5 Orthophoto of experimental area

Multi-images block adjustment experiment was carried out with different layout scheme of GCPs. The positioning accuracy was evaluated using CPs (Check Points), the results using the different number of GCPs are shown Table 4. In light of the requirement of 1:2,000 3D mapping specification, the plane accuracy is up to $1.75 \mathrm{~m}$ and elevation accuracy is up to $1 \mathrm{~m}$. The optimal GCPs layout scheme is usage of six GCPs to achieve multi-images block adjustment, as shown in Table 4 .

Table 4. Accuracy elevation with the layout scheme of GCPs

\begin{tabular}{|c|c|c|c|c|c|}
\hline \multicolumn{2}{|c|}{ Number } & \multicolumn{4}{c|}{ Accuracy(m) } \\
\hline \multirow{2}{*}{ GCPs } & \multirow{2}{*}{ CPs } & \multicolumn{2}{|c|}{ Plane } & \multicolumn{2}{c|}{ Elevation } \\
\cline { 3 - 6 } & & RMS & Max & RMS & Max \\
\hline 15 & 8 & 0.151 & 0.252 & 0.178 & -0.295 \\
\hline 12 & 11 & 0.151 & 0.273 & 0.172 & -0.299 \\
\hline 11 & 12 & 0.173 & 0.328 & 0.178 & -0.304 \\
\hline 9 & 14 & 0.182 & 0.351 & 0.200 & -0.322 \\
\hline 8 & 15 & 0.201 & 0.406 & 0.231 & 0.371 \\
\hline 7 & 16 & 0.184 & 0.391 & 0.196 & -0.329 \\
\hline 6 & 17 & 0.216 & 0.461 & 0.231 & 0.378 \\
\hline 5 & 18 & 0.263 & 0.808 & 0.368 & 1.331 \\
\hline 3 & 20 & 0.299 & 0.832 & 0.371 & 1.380 \\
\hline
\end{tabular}

Finally, the 3D mapping products were generated by the miniSAR mapping software, as showed in in Figure 6. The precision of DOM, DEM and DLG product meets the precision

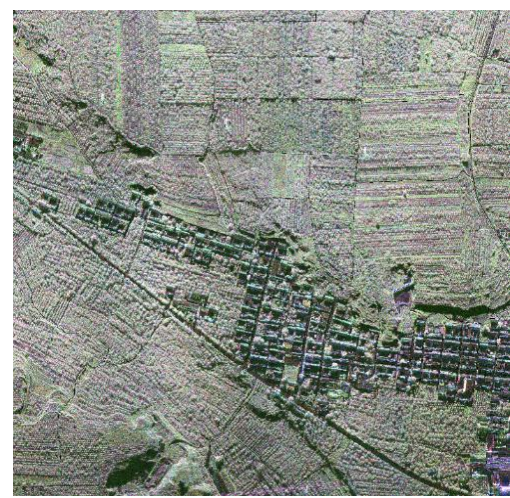

(a) DOM product 


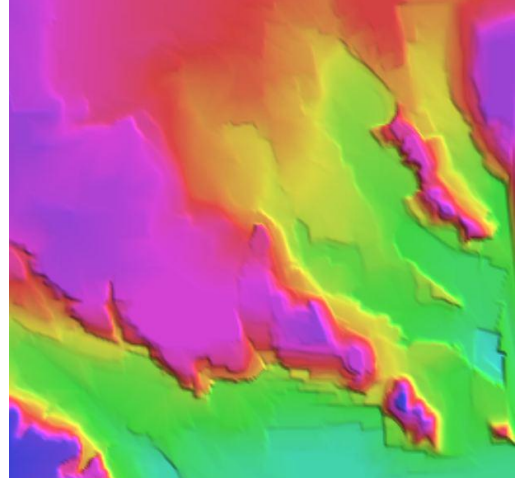

(b) DEM product

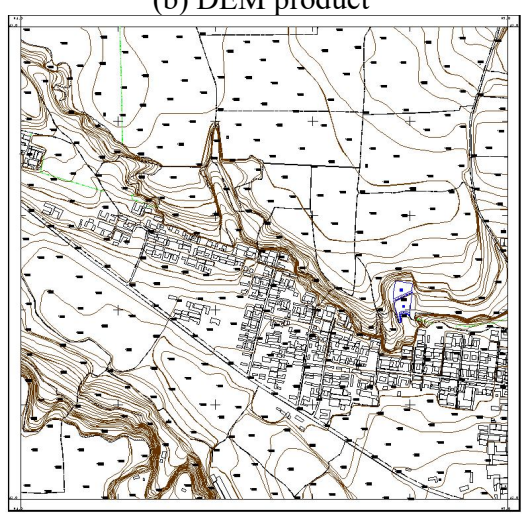

(c) DLG product

Figure 6 1:2000 3D mapping products of experimental area of 1:2000 mapping specification.

\subsection{Coastal Zone Monitoring}

In 2016, coastal zone monitoring campaign was implemented in lingshui county, Hainan province, China. Full polarimetric SAR image of Hailing island of was acquired as shown in Figure 7.

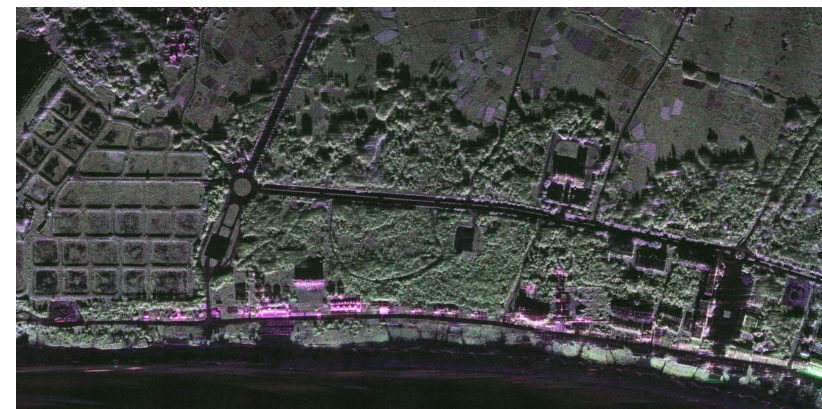

Figure 7. Full polarimetric SAR image of Hailing island

Coastal zone was easily extracted by dimensionality reduction and fusion of multi-Features, Figure 8 show the extraction result of coastal zone. As can be seen from Figure 8, the main type of land cover in the coastal zone is low shrubs.

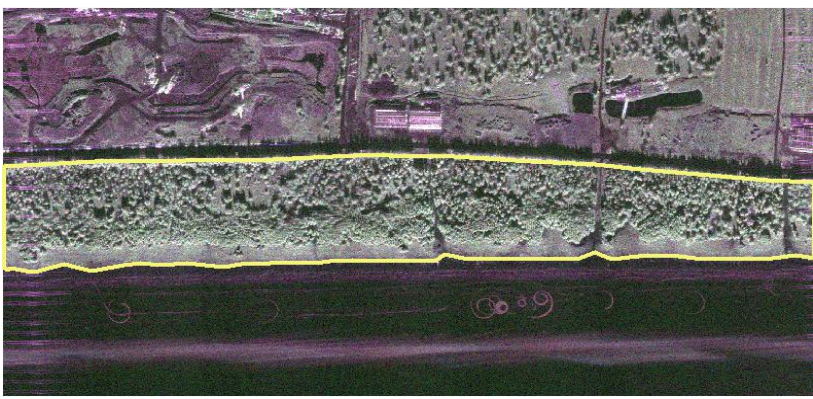

Figure 8. The extraction result of coastal zone of Hailing island

In addition, other type of land cover, e.g., coastal farms, was also extracted in similar way, as shown in Figure 9.

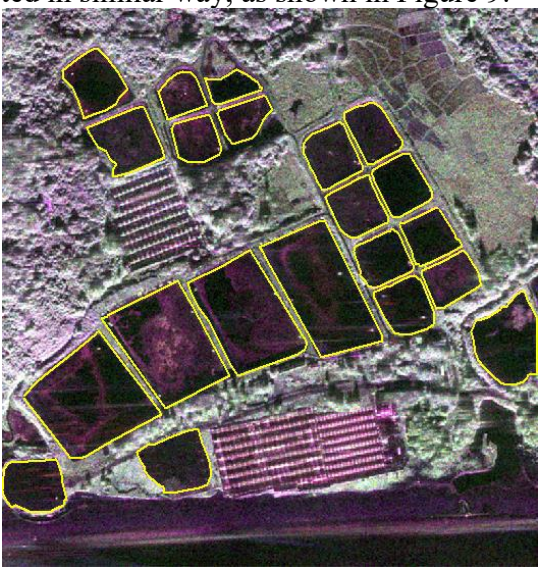

Figure 9. The extraction result of coastal farms of Hailing island

\section{CONCLUSION}

MiniSAR mapping technique is regarded as an effective sensing tool that offers all-weather, day and night operation in the fields of topographic mapping and emergency response. UAV platform enables rapid deployment and flexible data acquisition only by remote control or onboard computers. Therefore, the polarimetric miniSAR system has become a high priority for earth observation. Using state-of-the-art miniSAR techniques, the mapping system has been developed and applied to large scale 3D mapping and coastal zone monitoring. In the foreseeable future, the polarimetric miniSAR system will play a critical role in landscape mapping and emergence response.

\section{ACKNOWLEDGEMENTS}

The authors would like to thank the collaboration, support and help provided by Aerospace Information Research Institute, Chinese Academy of Science and Wuhan University. This work was supported by the National Key Research and Development Program of China, No. 2018YFF0215303 and 2017YFB0503004.

\section{REFERENCES}

Edwards, M., Madsen, D., Stringham, C., Margulis A., Wicks, B., Long, D. G., 2008. MicroASAR: A Small, Robust LFM-CW SAR for Operation on UAVs and Small Aircraft, Proceedings of IGARSS (International. Geoscience and Remote Sensing Symposium), Boston, MA, USA, pp.514-517.

Zaugg, E., Edwards, M., Margulis, A., 2010. The SlimSAR: A small, multi-frequency, Synthetic Aperture Radar for UAS operation, 2010 IEEE International Radar Conference, 10-14 May 2010, USA, pp.277-282.

Zaugg, E., Long, D., Edwards, M., etc, 2010. Using the MicroASAR on the NASA SIERRA UAS in the Characterization of Arctic Sea Ice Experiment, 2010 IEEE International Radar Conference, 10-14 May 2010, USA, pp.271-276.

Cheng, C.Q., Zhang, J.X., Deng, K.Z., Zhang, L., 2010. A new rigorous sensor model for radar imagery based on exterior 
orientation elements, ISPRS ARSS, 5-7 July 2010, Vienna, Austria, vol. XXXVIII, part 7B, pp.127-131.

Meier, E., Frei, U., Nüesch, D., 1993. Precise terrain corrected geocoded images. Karlsruhe, Wichmann.

Shi, L., Zhang L., Zhao L., etc, 2013. The Potential of Linear Discriminative LaplacianEigenmaps Dimensionality Reduction in Polarimetric SAR Classification for Agricultural Areas, ISPRS Journal of Photogrammetry and Remote Sensing, pp.124-135. 